\title{
OPTIMAL TWO-PART TARIFFS IN A MODEL \\ OF DISCRETE CHOICE*
}

\author{
by \\ Bruno De Borger \\ University of Antwerp (UFSIA)
}

\begin{abstract}
:
In this paper we study welfare-optimal two-part tariffs in a model of discrete choice. The determinants of the sign and magnitude of the fixed and variable tax are analysed in terms of the nature and degree of consumer heterogeneity, the price sensitivities of participation probabilities and conditional demands, and the nature of distributional concerns. In the absence of distributional concerns, the model generalises the 'corrected' Ramsey rule for the variable tax and it shows that, in the absence of collection or fixed participation costs, the fixed fee may be negative. The conditions that determine the sign of the participation fee turn out to have an intuitive economic interpretation. Finally, the implications of distributional concerns for the optimal fixed and variable taxes are analysed.
\end{abstract}

JEL classification codes: H21, H23

Key words: discrete choice, optimal taxation, two-part tariff.

*Please send all correspondence to Bruno De Borger, University of Antwerp (UFSIA), Prinsstraat 13, B-2000 Antwerp, Belgium. Alternatively, fax to (32 3) 2204420 or E-mail to bruno.deborgerpufsia.ac.be.

\section{Introduction}


It has been pointed out in the literature that nonlinear taxation and pricing of public services may be useful in enhancing both the efficiency and the distributional effectiveness of the public sector. Early studies have shown that a budget-constrained public authority may improve welfare by appropriate use of two-part tariffs (see, e.g., $\mathrm{Ng}$ and Weisser (1974), Leland and Meyer (1976), Spence (1980), Schmalensee (1981)). These models typically assume a continuum of consumers characterised by a one-dimensional taste parameter. They show that the fixed participation fee will be nonnegative and that the relation between the variable fee and marginal production cost depends on consumer heterogeneity, i.e., on consumption of the marginal consumer relative to average consumption ${ }^{1}$. More recently, it has been shown that the variable component of the two-part tariff can be interpreted as a 'corrected' Ramsey price, where the correction term again depends on the relation between marginal and average consumption. (Brown and Sibley (1986), Laffont and Tirole (1993)). More sophisticated pricing structures have been studied in a voluminous literature, stressing the role of self-selection and asymmetric information and the necessity of nonlinear prices for efficient regulatory policies. For surveys of these developments and applications to public utility regulation, see Brown and Sibley (1986), Breautigam (1989) and Wilson (1993). Finally, the potential role of nonlinear prices in the presence of distributional concerns and asymmetric information has been studied by, e.g., Sharkey and Sibley (1993) and Cremer and Gahvari $(1995)^{2}$. They show that when consumers differ in taste nonlinear prices still have a redistributive role, even under circumstances where they are not needed for efficiency purposes.

\footnotetext{
${ }^{1}$ If fixed production costs are associated with participation, Schmalensee (1981) points out that the fixed fee may be above or below the corresponding fixed cost. The sign of the net participation fee depends on substitution effects in demand and heterogeneity among consumers. ${ }^{2}$ An early paper by Feldstein (1972) analysed two-part tariffs in the presence of distributional concerns. However, he assumed that participation was fixed, which effectively makes the fixed fee analogous to a lump-sum tax that cannot be avoided.
} 
Not surprisingly, governments frequently use nonlinear schedules in pricing publicly provided services and in designing indirect tax structures. Examples include pricing of telephone services, electricity, water and gas utilities, railroad services, and public parking facilities (see, e.g., Damus (1982), Phlips (1983), Sibley and Brown (1986), Glazer and Niskanen (1992), and Wilson (1993)). Although in practice nonlinear pricing ranges from two-part tariffs to highly sophisticated pricing structures, including declining block tariffs (Phlips (1983) and optional tariffs (Train, McFadden and Ben-Akiva (1987)), two-part tariffs are an important ingredient of such schedules. Interestingly, two-part tariffs are also often imposed on durable goods in cases where ownership and consumption can be separately priced or taxed. Taxation of cars provides a stylised example. In many European countries, car owners pay an annual vehicle tax as well as a 'user' tax, mainly in the form of a fuel tax (see ACEA (1996)) ${ }^{3}$.

In this paper we study welfare-optimal two-part tariffs within the framework of a model of discrete choice. Somewhat surprisingly, the literature has not exploited the potential of discrete choice models for the analysis of two-part tariffs. Such an approach seems to be highly relevant for several reasons. First, two-part tariffs are often imposed on durable goods where at the individual level consumers are faced with an explicit discrete choice. Again, taxation of cars is an obvious example: consumers decide whether or not to own a car, and they decide on the intensity of car usage. At the level of a typical consumer discrete choice models provide an integrated framework that explicitly captures the interactions between the ownership decision and the conditional demand for the good (see, e.g., Small and Rosen (1981), Anderson, de Palma and Thisse (1993)). Second, since the introduction of a two-part tariff conceptually separates the participation and consumption decisions, discrete choice models provide a natural tool for the

\footnotetext{
${ }^{3}$ Again, more sophisticated tax structures are often used. For example, a highly nonlinear tax structure on owner-occupied housing is implied by, among other factors, the partial tax deductibility of intrest payments (Rosen (1979)).
} 
analysis of two-part tariffs even on markets where consumers do not face an explicit ownership decision. Since consumers decide on whether to buy the product and on how much to consume, the two-part tariff implicitly transforms the problem into a discrete choice issue ${ }^{4}$. Third, a discrete choice framework allows a fairly general and multi-dimensional description of consumer heterogeneity without substantially complicating the analysis. Although the intrinsic onedimensionality of tastes assumed in the literature may not be crucial for several of the qualitative conclusions obtained, a richer specification of heterogeneity among consumers does yield additional information ${ }^{5}$. Finally, the analysis of two-part tariffs in a discrete choice framework may be useful for applied optimal tax models: incorporating discrete choice components in numerical optimisation models may allow a more realistic treatment of taxation of ownership and use of specific durable goods such as, e.g., vehicles. Given the large and growing empirical literature on discrete choice behavior (see, e.g., Ben-Akiva and Lerman (1987), Börsch-Supan (1987), Train (1986), De Jongh (1990)), many of the parameters required to operationalise such models are available.

The purpose of the paper is to analyse welfare-optimal two-part tariffs for a government facing budgetary constraints in an explicit model of discrete choice, incorporating a quite general specification of consumer heterogeneity and allowing the government to have distributional preferences. The analysis highlights the role of both the nature and the degree of heterogeneity across consumers, of the distribution of price sensitivities across consumers, of the severity of budgetary constraints, and of the nature and degree of distributional preferences of the government. It both generalises and extends the existing literature. In the absence of distributional concerns the optimal tax rule for the variable fee can be interpreted as a

\footnotetext{
${ }^{4}$ In this sense, as noted by a referee, there is no substantial difference between an explicit discrete choice market and other markets with two-part tariffs.

${ }^{5}$ See Wilson (1993, p. 194-200) on the difficulties associated with multiple dimensions of consumers' types for pricing.
} 
multidimensional generalisation of results in Schmalensee (1981), Brown and Sibley (1986), and Laffont and Tirole (1993). Moreover, it is shown that in the absence of collection or other fixed costs associated with participation, the fixed fee may be negative. The conditions that determine the sign of the participation fee turn out to have a surprisingly simple economic interpretation. The analysis generalises the 'corrected' Ramsey rule (Laffont and Tirole (1993)) and provides an explicit and intuitive relation between the Ramsey correction term and the sign of the (net of collection costs) fixed fee. Finally, if the government does care about distribution, the optimal two-part tariff trades off demand heterogeneity, price sensitivities, and distributional concerns.

The paper is organised as follows. The structure of the model is developed in Section 2. We formulate the welfare-optimal two-part tariff under a budgetary restriction within the framework of a logit model of discrete choice, taking account of consumer heterogeneity and distributional concerns. The logit model was used because it has been widely used in theoretical and empirical work and, more importantly, because it yields a closed-form expression for the participation probabilities and for the welfare function associated with the optimal tax problem. However, it will be shown that the results are generally applicable to other discrete choice models as well. In Section 3 we derive the optimal two-part tariffs, interpret the results, compare with previous results in the literature and discuss some implications. In Section 4 we conclude with a brief summary of our findings.

\section{Two-part tariffs in a logit model of discrete choice}

We consider the simplest possible setup for the study of optimal two-part tariffs in a discrete choice framework. Suppose consumers care about just two goods. The first good (x) is subject to a two-part tariff, the second one (z) is treated as numeraire. The demand for $\mathrm{x}$ involves 
both a discrete choice (consume the good or not consume it at all) and a continuous choice (desired consumption levels). It can be interpreted either as a government-provided service or as a private good where the government imposes taxes on both ownership and intensity of use. For concreteness sake, we will cast the model in terms of a private good where consumption involves a decision with respect to ownership of a durable good and, conditional on the ownership decision, determination of desired consumption levels. The demand for kilometers traveled by car provides a stylised example. Consumers decide whether or not to own a car, and they determine the optimal mileage. Both ownership and use are taxed.

Let there be $\mathrm{H}$ consumer types, each type $\mathrm{h}$ consisting of $\mathrm{n}^{\mathrm{h}}$ people. Following the discrete choice literature, let us assume that the indirect utility $v_{i}^{h}$ for a randomly chosen individual in group $\mathrm{h}$, conditional on his choice of alternative $\mathrm{i}(\mathrm{i}=1$ if he decides to own the durable good, $\mathrm{i}=2$ otherwise) consists of a deterministic component $V_{i}^{h}$ plus a random term $u_{i}^{h}$, i.e., $v_{i}^{h}=V_{i}^{h}+u_{i}^{h}$. The stochastic component may, e.g., reflect unobservable taste differences between individuals (see, e.g., Manski (1977) and Anderson et al. (1993) on various possible interpretations). Denoting the fixed and variable components of the two-part tariff by $\mathrm{F}$ and $\mathrm{p}$, respectively, the deterministic component of indirect utility for an individual deciding to consume a positive amount of the good (i.e., an owner of the durable good) can be defined as the solution to a standard utility maximisation problem:

$$
V_{1}^{h}\left(p, y^{h}-F\right)=\operatorname{Max}_{x^{h}, z^{h}} \quad U^{h}\left(x^{h}, z^{h}\right) \text { s.t. } p x^{h}+F+z^{h}=y^{h}
$$


where $U^{h}\left(x^{h}, z^{h}\right)$ is the individual's direct utility function, and $y^{h}$ his income ${ }^{6}$. Utility in the alternative case is denoted by $V_{2}^{h}\left(y^{h}\right)$; it corresponds to the level of well-being at zero consumption of x. Following Small and Rosen (1981) it is assumed that this utility level is finite.

A randomly chosen individual of type $h$ will select alternative $i$ for which $v_{i}^{h}=\underset{i}{\operatorname{Max}}\left(v_{1}^{h}, v_{2}^{h}\right)$. Assuming the $u_{i}^{h}$ to be i.i.d. double exponential then yields the well-known logit model of discrete choice ${ }^{7}$. The probability $\pi^{h}$ of choosing alternative 1 (i.e., to own the durable good) is then given by

$$
\pi^{h}\left(p, y^{h}-F\right)=\frac{e^{\left(\frac{V I^{h}}{\mu^{h}}\right)}}{e^{\left(\frac{V_{1}^{h}}{\mu^{h}}\right)}+e^{\left(\frac{V^{h} 2}{\mu^{h}}\right)}},
$$

where $\mu^{h}$ is a scale parameter of the joint distribution of the error terms. It reflects the variance of preferences of consumers of type h over the two alternatives. Expected demand for the first good in the population is given by

$$
X=\sum_{h} n^{h} \quad \pi^{h}\left(p, y^{h}-F\right) \quad x^{h}\left(p, y^{h}-F\right)
$$

where $x^{h}($.$) is the conditional individual demand function for the first good; it can be derived$ from $V_{1}^{h}($.$) by Roy's identity. Note that both x^{h}($.$) and \pi^{h}($.$) depend on \mathrm{p}$ and F.

The government is assumed to determine $\mathrm{F}$ and $\mathrm{p}$ so as to maximise welfare subject to a budgetary restriction. As a measure of individual welfare associated with the available options we use the expected value of maximum utility (see, e.g., Ben Akiva and Lerman (1987)). The

\footnotetext{
${ }^{6}$ Note that owning the first good may in reality require a fixed annual expense (e.g., the annual cost of owning a car). Since incorporating this fixed cost in no way affects the optimal two-part tariff it is ignored in the above formulation.

${ }^{7}$ The main advantage of the logit model is that both the probability of ownership and the welfare function to be maximised can be written as closed-form expressions. This highly simplifies the analysis. However, it is shown in Appendix C below that all results presented in the next section hold for discrete choice models in general, including those that do not yield closed-form expressions for probabilities and welfare.
} 
logit specification implies that, for a consumer of type $h$, this expected value can be written as (see , e.g., Anderson, de Palma, and Thisse (1993))

$$
\mu^{h} \boldsymbol{\Psi} \mathbf{p}\left(\frac{V_{1}^{h}}{\mu^{h}}\right)+\exp \left(\frac{V_{2}^{h}}{\mu^{h}}\right)
$$

The government's budget constraint is defined in terms of expected revenues and costs. Assuming a constant producer price equal to marginal production cost c, it can be written as

$$
(p-c) n_{n}^{h} \pi^{h} x^{h} \mathbf{k}+F n^{h} \pi^{h} \mathbf{k}=R
$$

or

$$
(p-c) X+F N=R
$$

where $\mathrm{X}$ and $\mathrm{N}$ are total expected demand and total number of owners of the durable good, respectively, and $\mathrm{R}$ is required net revenue, assumed to be positive.

\section{Optimal two-part tariffs}

In this section we first formulate the optimal taxation problem and derive the optimal pricing rules. We then analyse the economic characteristics of the optimal two-part tariff both in the absence and in the presence of distributional considerations. Finally, we discuss some extensions and potential applications.

\subsection{Derivation of the optimal two-part tariff}

In view of the discussion in the previous section, the problem of determining the welfareoptimal two-part tariff can be formulated as follows: 


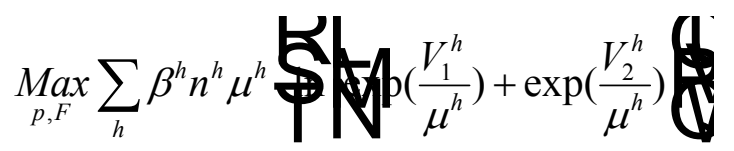

$$
\begin{aligned}
& \text { s.t. } \quad(p-c) \overline{\mathbf{n}} n^{h} \pi^{h} x^{h} \mathbf{k} F \mathbf{5} n^{h} \pi^{h} \mathbf{k}=R \text {, }
\end{aligned}
$$

where the $\beta^{h}$ reflect distributional concerns of the government. Denoting the multiplier associated with the budget constraint by $\lambda$, it is shown in Appendix A that the first-order conditions with respect to $\mathrm{p}$ and $\mathrm{F}$ can be manipulated to yield

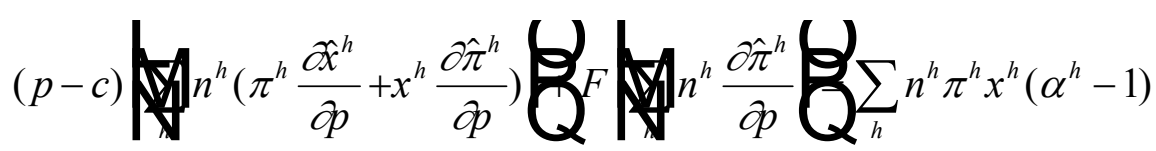

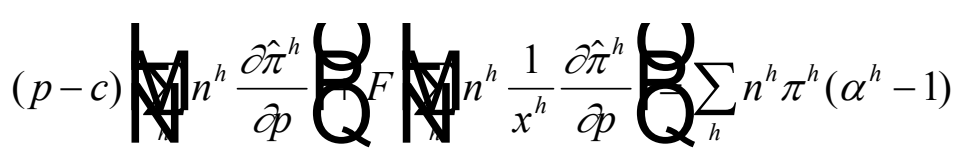

where $\hat{x}^{h}$ and $\hat{\pi}^{h}$ are the compensated conditional demand function and the compensated choice probability (see Small and Rosen (1981)), respectively, and

$$
\left.\alpha^{h}=\frac{\beta^{h}}{\lambda} \frac{\partial V_{1}^{h}}{\partial y^{h}}+\frac{1}{\pi^{h}} \mathbf{W}-c\right)\left(\pi^{h} \frac{\partial x^{h}}{\partial y^{h}}+x^{h} \frac{\partial \pi^{h}}{\partial y^{h}}\right)+F \frac{\partial \pi^{h}}{\partial y^{h}}
$$

The $\alpha^{h}$ measure the net marginal social utility of income (see Diamond (1975)) for owners of the durable good in the various groups $h$, normalised by the appropriate ownership probability (see Appendix A for more details).

There are different ways to proceed. Defining $\rho^{h}=n^{h} \frac{1}{x^{h}} \frac{\partial \hat{\pi}^{h}}{\partial p}$, writing (4) and (5) in matrix notation and solving the resulting system using Cramer's rule yields 
$p-c=\frac{1}{D}$

$F=\frac{1}{D} \frac{1}{2} n^{h}\left(\pi^{h} \frac{x^{h}}{\partial p}+x^{h} \frac{\partial \hat{\pi}^{h}}{\partial p}\right)$ Q

where

$$
D=\mathbb{W} n^{h}\left(\pi^{h} \frac{\partial^{h}}{\partial p}+x^{h} \frac{\partial \hat{\pi}^{h}}{\partial p}\right) \text { Liy } \rho^{h} \text { Q }
$$

is the determinant associated with equation system (4)-(5). It is shown in Appendix B that $\mathrm{D}>0$. To further simplify expressions (7) and (8), note that we can write

$$
\sum_{h} n^{h} \pi^{h}\left(\alpha^{h}-1\right)=N(\bar{\alpha}-1)
$$

where $\bar{\alpha}=\frac{\sum_{h} n^{h} \pi^{h} \alpha^{h}}{N}$ is 'average' marginal social utility. Using this result in (7) and (8) yields, after some simple manipulations

$$
\begin{aligned}
& p-c=\frac{1}{D} \boldsymbol{\Psi}\left(\sum_{h} \rho^{h}\right)-B[N(\bar{\alpha}-1)] \\
& F=\frac{1}{D} \boldsymbol{\Psi}[N(\bar{\alpha}-1)]-\gamma\left(\sum_{h} \rho^{h} x^{h}\right) \boldsymbol{V}
\end{aligned}
$$

where

$$
A=\sum_{h} n^{h} \pi^{h} \frac{\partial \hat{x}^{h}}{\partial p}+\sum_{h} n^{h} \frac{\partial \hat{\pi}^{h}}{\partial p}\left(x^{h}-\frac{X}{N}\right)
$$




$$
\begin{aligned}
& B=\sum_{h} n^{h} \frac{\partial \hat{\pi}^{h}}{\partial p}\left(1-\frac{X}{x^{h} N}\right) \\
& \gamma=\sum_{h} n^{h} \pi^{h} x^{h}\left(\alpha^{h}-\bar{\alpha}\right) .
\end{aligned}
$$

Equations (9) and (10) are the basic expressions describing the optimal two-part tariff. As they play a crucial role in the following discussion, it is instructive to briefly consider the economic meaning and the sign of the terms A, B, and $\gamma$. First consider B. It describes the nature and the degree of demand heterogeneity between groups. If there is no demand heterogeneity (i.e., $x^{h}=\mathrm{X} / \mathrm{N}$ for all $\mathrm{h}$ ) it immediately follows that $\mathrm{B}=0$. Alternatively, $\mathrm{B}$ can be positive or negative depending on the nature of heterogeneity. If the ownership probabilities are generally less price sensitive for groups with above average conditional demands then B is positive. Under these circumstances the value of B rises when more heterogeneity exists between groups. If on the contrary the entry probabilities of high demand groups are more price sensitive then B will be negative.

Term A is composed of two parts. The first one is a weighted sum of Slutsky terms and is, therefore, always negative. The second term again relates to the degree of heterogeneity with respect to the choice probabilities. The sign of A therefore depends on the type of heterogeneity and the importance of the Slutsky effects. Interestingly, however, the conditions for A to be positive or negative turn out to have a simple economic interpretation. To see this, consider average consumption by owners of the good, $\bar{x}=\mathrm{X} / \mathrm{N}$. Differentiating average consumption with respect to the variable price $p$ yields

$$
\frac{\partial x}{\partial p}=\frac{N \sqrt[W]{\mid} n^{h}\left(\pi^{h} \frac{\partial^{h}}{\partial p}+x^{h} \frac{\partial \hat{\pi}^{h}}{\partial p}\right) Q^{2} n^{h} \frac{\partial \hat{\pi}^{h}}{\partial p} \mid}{N^{2}}
$$


Slightly rearranging and using the definition of A implies

$$
\frac{\partial x}{\partial p}=\frac{A}{N}
$$

In other words, the sign of A reflects the impact of a price increase on average consumption. If a price increase reduces average consumption then $\mathrm{A}<0$. If the opposite holds $\mathrm{A}>0$.

Finally, it is clear that $\gamma$ captures information on the distribution of the net marginal social utilities, and therefore on the degree and the nature of distributional concern by the government. If groups with low expected demands are assigned above average net marginal social utilities then $\gamma$ is negative. It is positive if the opposite holds.

Before turning to a more detailed interpretation of the basic results, note that (9) and (10) immediately allow two general conclusions. A first observation is that marginal cost pricing plus a positive ownership fee is optimal under two sets of conditions. One is the case where there is no heterogeneity in demand between groups, i.e., $x^{h}=\mathrm{X} / \mathrm{N}$ for all $\mathrm{h}$. It then immediately follows that $\mathrm{A}<0$ and $\mathrm{B}=0$. Moreover, simple algebra shows that in that case $\gamma=0$, so that (9) and (10) imply $\mathrm{p}=\mathrm{c}$ and $\mathrm{F}>0$. This confirms earlier results of, e.g., $\mathrm{Ng}$ and Weisser (1974), Spence (1980) and Schmalensee (1981). Alternatively, assume that the ownership probabilities are totally price insensitive. This also implies marginal cost pricing plus a positive fixed fee to satisfy the budget restriction. Second, equations (9) and (10) identify all key determinants of the sign and magnitude of the optimal variable and fixed taxes: the nature and degree of heterogeneity between groups (captured by A and B), the stringency of the budget constraint, and the nature and degree of distributional concern of the government (the latter two effects reflected in $\gamma$ ).

\subsection{The optimal two-part tariff: efficiency issues}


In order to facilitate comparison with previous results in the literature we first focus on efficiency issues by ignoring income effects and distributional concerns. Specifically, assume $\frac{\partial x^{h}}{\partial y^{h}}=\frac{\partial \pi^{h}}{\partial y^{h}}=0$ and let private marginal social utilities be constant for all groups, i.e., $\beta^{h} \frac{\partial V_{1}^{h}}{\partial y^{h}}=\mu$ for all $\mathrm{h}$, where $\mu$ is the common marginal social utility of income. Simple algebra shows that these assumptions imply $\gamma=0$ and $N(\bar{\alpha}-1)=N\left(\frac{\mu-\lambda}{\lambda}\right)$, so that (9) and (10) reduce to

$$
\begin{aligned}
& p-c=\left(\frac{\lambda-\mu}{\lambda}\right) \frac{N}{D} B \\
& F=-\left(\frac{\lambda-\mu}{\lambda}\right) \frac{N}{D} A
\end{aligned}
$$

Assuming that the shadow cost of public funds exceeds the marginal social utility of income to reflect the presence of distortionary taxes elsewhere in the economy, it follows that the sign of (p-c) and $\mathrm{F}$ is uniquely determined by the signs of B and A, respectively. First consider the variable tax $(\mathrm{p}-\mathrm{c})$. Its sign is uniquely determined by the heterogeneity in the sensitivity of the entry probabilities with respect to price changes. Suppose, for example, that the probability of participation is generally more price sensitive for low-demand than for high-demand groups, so that $\mathrm{B}>0$. It follows from (11) that the variable tax will be positive, $(\mathrm{p}-\mathrm{c})>0^{8}$. Alternatively, a variable subsidy is optimal if participation by high demand groups is generally more price sensitive than for low demand groups.

Note that the optimal variable tax rule (11) can be interpreted as a 'multidimensional' generalisation of earlier results in , e.g., $\mathrm{Ng}$ and Weisser (1974), Schmalensee (1981), and 
Laffont and Tirole (1993). These models typically assumed a one-dimensional distribution of consumer types and find positive variable taxes as long as marginal is less than average consumption. In the current paper the sign of the variable tax depends on the correlation between consumption and the price sensitivities of participation. The tax will be positive if a price reduction has generally a stronger impact on participation by low demand groups, so that the induced extra consumption more than proportionally comes from small demanders.

The fixed fee F may be positive or negative depending on the sign of the term A (see (12)). As long as a variable price increase reduces average consumption levels $\mathrm{X} / \mathrm{N}$ (or alternatively, as long as the sum of Slutsky effects dominates the heterogeneity term) we have $\mathrm{A}<0$, and therefore $\mathrm{F}>0$. However, if compensated price elasticities are generally small and are dominated by heterogeneity so that price increases yield higher average consumption levels, it is optimal to subsidise entry. This shows that, in the absence of fixed participation costs or collection costs associated with $\mathrm{F}$, the participation fee can be negative ${ }^{9}$. This possibility was excluded in earlier work on two-part tariffs (Ng and Weisser (1974), Schmalensee (1981), Brown and Sibley (1986)) because in a deterministic framework a participation subsidy would induce everyone to participate, even those that do not plan any consumption. The randomness in utility that underlies discrete choice models invalidates this universal participation argument. Of course, in practice there will be a limit to the magnitude of a fixed subsidy, since for increasingly large subsidies the probability of nonparticipation eventually does go to zero. Limited subsidies are however quite possible.

\footnotetext{
${ }^{8}$ Inspection of the terms B and D show that in this case the variable price converges to marginal cost $\mathrm{c}$ under three sets of conditions: if conditional demand elasticities become extremely large, if the price sensitivity of the probabilities of entering the market approach zero, and if heterogeneity becomes extremely small so that B converges to zero.

${ }^{9}$ Note that the introduction of fixed participation costs or collection costs associated with participation does not alter the general rule: the sign of the (net of fixed participation costs) entry fee is uniquely determined by the sign of the term A. This generalises findings in Schmalensee (1981). He indicates that, depending on the relation between marginal and average consumption and the magnitude of substitution effects, the fixed fee may be above or below the fixed cost. Our findings extend his findings to the case of zero participation costs and provide an economically appealing interpretation of the sign of the (net of fixed costs) fee.
} 
Expressions (11) and (12) clarify the role of the heterogeneity terms A and B for the signs of the variable and fixed tax components. Further insight can be gained by slightly manipulating (11). Defining the aggregate price elasticity of demand as

$$
e_{p}=\frac{\partial X}{\partial p} \frac{p}{X}=\frac{p}{X}
$$

and using this definition in (11) we obtain after slight manipulations

$$
\frac{p-c}{p}=\left(\frac{\lambda-\mu}{\lambda}\right) \frac{1}{\left|e_{p}\right|} \frac{N\left(\sum_{h} \rho^{h} x^{h}\right) A}{D X}
$$

Equation (13) is a 'corrected' Ramsey form that is, at least structurally, similar to Laffont and Tirole (1993, p. 148) and Wilson (1993, p. 138). In the latter models the correction term is less that one as long as marginal is less that average consumption. In the model considered in this paper the magnitude of the Ramsey correction term in (13) depends on the distribution of the price sensitivities of the participation probabilities and on the impact of price increases on average consumption, as captured by the term A. Interestingly, note that the sign of A simultaneously determines the sign of the fixed fee and the relation of the variable tax to the standard Ramsey case. This makes sense. Indeed, if $A=0$ then (12) implies that the fixed fee would be zero and (13) reduces to the standard Ramsey rule for the variable tax. A positive fixed fee allows the variable tax to be reduced as compared to the Ramsey case, and a subsidy for participation is compensated by an increase in the variable tax above the Ramsey tax.

Based on the above discussion Table 1 summarises the relation between the nature of heterogeneity and the structure of the optimal two-part tariff. First, if $\mathrm{B}>0$ and $\mathrm{A}<0$ both tax components are positive, and the variable tax is less than the Ramsey tax (in the sense that the Ramsey correction term in (13) is less than one). Second, if $\mathrm{B}>0$ and $\mathrm{A}>0$ we find a negative 
fixed fee combined with a positive variable tax that actually exceeds the standard Ramsey tax; the correction term is larger than one. Intuitively, given the nature of heterogeneity considered, the negative fee $\mathrm{F}$ affects ownership by low demanders much more than ownership by high demand groups. To attain the required budget, it is necessary to raise the variable price $\mathrm{p}$ above the standard Ramsey tax to compensate for the ownership subsidy. Since the price increase actually raises average consumption extra revenues are relatively easy to generate. Welfare improves as the effects of subsidising $\mathrm{F}$ more than outweigh the welfare reduction due to the increase in the variable tax. Third, suppose that the entry probabilities and conditional demands of high demand groups are generally more price sensitive, so that B and A are both negative. It is then optimal to combine a fixed tax with a variable subsidy. Intuitively, increasing the fee implies that mainly high demanders are lost; this reduces the budgetary effects of subsidising use of the good. The welfare effect of the small increase in $\mathrm{F}$ required to finance the variable subsidies is dominated by the effect of the price subsidy itself.

\section{INSERT TABLE 1 ABOUT HERE}

The above results identified the correlation between conditional demands and the price sensitivity of participation and the effect of a price increase on average consumption levels as important determinants of the fixed and variable taxes. In principle, information on these effects on real markets can be obtained from empirical research. Of course, the theoretical model was restricted to one good and therefore ignored the possible existence of close substitutes and the corresponding cross-price effects in optimal tax design ${ }^{10}$. With this caveat in mind it is

\footnotetext{
${ }^{10}$ Extending the theoretical model to multiple discrete options is conceptually straightforward but analytically cumbersome, unless some strong assumptions are imposed on cross-price effects. Intuitively, optimal two-part tariffs would depend on the complete distribution of both own and cross-price effects.
} 
nevertheless interesting to see whether empirical evidence allows us to determine the sign of $\mathrm{A}$ and B for some specific markets. Probably the best available evidence is related to the car market. First, both De Jongh (1990) and Train (1986) implicitly contain interesting information on the correlation between price sensitivities of the ownership probabilities and conditional demands. The former analyses the aggregate demand for cars and associated mileage, where the ownership decision is specified as a logit model of discrete choice. He finds strong evidence that ownership probabilities are less price elastic for high income consumers and that high income groups have a higher conditional demand for mileage than lower income people. Together these findings imply negative correlation between ownership probabilities and conditional demands (i.e., B $>0$ ). The model described in Train (1986, chapter 8) analyses ownership decisions (how many vehicles to own), which type of vehicle to own (class and vintage), and mileage. His results suggest that the ownership probability (both at the aggregate level and for particular car types) is more price sensitive with respect to purchase price for low income families. Since average mileage rises with income this could again be interpreted as evidence of a negative correlation between participation and demands ${ }^{11}$. De Jongh (1990) also observes that car ownership is more price sensitive with respect to fixed car costs than with respect to variable price, whereas the opposite holds for car use. More importantly; the estimated elasticities imply a higher price sensitivity of total mileage than of total car ownership. As a consequence, his estimates imply that variable price increases lead to a reduction in average mileage, which is precisely the condition for $\mathrm{A}<0$. His reported simulation results imply this elasticity to be about 0.27. Finally, both Small (1983) and Train (1986) imply nontrivial compensated price elasticities of car mileage (conditional on ownership), a finding that is again consistent with $\mathrm{A}<0$. Ignoring

\footnotetext{
${ }^{11}$ Of course, price sensitivities with respect to purchase price do not perfectly capture elasticities with respect to annual fixed costs due to differences in replacement behavior and implicit discount factors used.
} 
distributional issues, all the above evidence indicates the optimality of positive variable as well as fixed taxes, even in the absence of externalities.

In situations where consumers face discrete choices among a number of highly substitutable alternatives one expects the optimal two-part tariff for any given option to strongly depend on cross-price elasticities as well as on own price effects. Generalising the theoretical model to deal with these cases is not analytically attractive, so that numerical optimisation techniques may have to be used to gain further insight. However, the important point is that the necessary information (e.g., own and cross-price elasticities of both ownership probabilities and conditional demands) required to calibrate discrete choice optimal taxation models can again be obtained from empirical analyses of discrete choice models. For example, in a study of choices between appliances, Dubin and McFadden (1984) report elasticities of the ownership probability of electric appliances with respect to the variable price of electricity of -0.45 , whereas the price elasticity of electricity demand conditional on the choice of electric appliances is about -0.3 . The lower elasticity of conditional demand suggests that, at constant prices of substitutes, an increase in electricity prices may actually increase average electricity consumption. Not surprisingly, however, cross-price effects are estimated to be substantial. In addition, several studies on the US telephone industry by Train, McFadden en Ben-Akiva (1987) and Train, Ben-Akiva and Atherton (1989) explicitly analyse consumer choices among many alternative service options. The former study reports elasticities of the choice probabilities of specific options with respect to the corresponding fixed charge that largely exceeds the price elasticity of the conditional demand for calls. Again, cross-price effects are found to be nontrivial. Moreover, the latter study explicitly tests for the validity of the hypothesis of single crossing of demands. Within the terminology of this paper, this is closely related to testing whether $\mathrm{B}>0$. They find that the condition is not generally satisfied, but that deviations are not severe. 


\subsection{Introducing distributional issues}

We now reintroduce possible income effects in demand and consider the case where the government does care about distribution. Introducing income effects complicates the algebra, but it is easily shown not to alter the conclusion that the signs of variable and fixed taxes are uniquely determined by the signs of the terms A and B. Distributional issues do make a difference, of course. Not surprisingly, the impact of distributional issues strongly depends on the nature of such concerns, i.e., on the correlation between net marginal social utilities and consumption. To fix ideas, let us initially assume that the net marginal social utilities of income $\alpha^{h}$ are generally higher for low-demand groups, so that $\gamma<0$. It may be the case that low demanders (i.e., groups with conditional demands $x^{h}$ below the average demand $\mathrm{X} / \mathrm{N}$ ) are generally associated with higher marginal social utilities. Alternatively, the induced tax revenues for the government may be higher for low demand groups due to larger marginal propensities to consume. Note that for many goods a negative $\gamma$ is not unreasonable (e.g., electricity, housing, etc.): demands typically increase with income and governments may well favor redistribution towards the poor.

To see the implications of distributional concerns of the type $\gamma<0$, again consider (9) and (10). It follows that, conditional on a given average net marginal social utility $\bar{\alpha}$, introduction of distributional preferences favoring low-demand groups reduces the optimal fixed fee $\mathrm{F}$ and increases the variable tax $(\mathrm{p}-\mathrm{c})$. Moreover, this finding is independent of the optimal price structure in the absence of distributional concerns. If in the reference case without distributional preferences both tax components are positive (case $\mathrm{B}>0$ and $\mathrm{A}<0$ ) it makes intuitive sense to 
reduce the fixed fee and to increase the unit tax. A lower fixed fee benefits low-demand groups, and the burden of the variable tax increase mainly falls on high-demand groups. Second, if in the reference case of zero distributional concern we have initially a fixed subsidy and a positive variable tax, distributional preferences in favor of low-demand groups imply an increase in the variable tax combined with an even larger fixed subsidy for the same reasons. Third, if initially the optimal price structure implies a variable subsidy and a fixed tax, redistrubution towards low demanders requires the reduction of the variable subsidy and the reduction of the fixed fee.

If distributional concerns are such that groups with high expected demands are associated with a larger net social marginal utility then $\gamma>0$. In that case the optimum moves in the other direction: lower variable and higher fixed taxes. Note that a positive $\gamma$ is not unreasonable in at least two types of situations. It is likely to hold for inferior goods under the assumption that governments favor redistribution towards the poor. To the extent that expected demand declines at high incomes, public transport may be a relevant example. There is quite some evidence that increasing income substantially reduces the probability of using public transport (see, e.g., Small (1983)). Alternatively, $\gamma>0$ may well hold for merit goods (culture, education) to the extent that the government wants to stimulate overall consumption by assigning larger welfare weights to high demanders. In both cases more distributional concern implies lower variable taxes and higher fixed fees, since the benefit of the lower variable tax largely goes to high demanders.

Apart from the recent studies by Sharkey and Sibley (1993) and Cremer and Gahvari (1995) that explicitly deal with the role of distributive pricing in the presence of asymmetric information, the only previous analysis of distributional issues for optimal two-part tariffs is an early paper by Feldstein (1972). He reports that the variable price component will be zero in the absence of distributional concerns and that, when distribution does matter, the sign of the 
variable tax is uniquely determined by the correlation between marginal social utilities and consumption. It will be positive as long as low demand groups are generally valued higher in the social welfare function. His model, however, implicitly assumes participation to be fixed, making the fixed fee analogous to a lump-sum tax that cannot be avoided. If participation is not independent of pricing and tax payments are conditional on participation, then the fixed fee is clearly not equivalent to a lump-sum tax, and Feldstein's conclusions are no longer valid. As shown before, even in the absence of distributional concerns the variable tax will be nonzero (see (9)). In the presence of distributional concerns, the sign and magnitude of the variable tax are the result of a careful trade-off between demand heterogeneity, variability in the ownership probabilities, and distributional concerns.

It is clear that one should be careful with the use of observed pricing policies to support a normative theory, because observed policies are unlikely to be the result of welfare optimisation. It is nevertheless interesting to point out that several observed pricing practices are at least broadly consistent with the results on distributive pricing. A first potential example again relates to the car market. To the extent that car use rises with income and ignoring cross-price effects, distributional concerns suggest lower fixed fees on cars highly bought by low demand customers, and a correspondingly higher tax on vehicle use. Although the problem is highly complicated due to cross-price effects, to the role of freight transport and the presence of external transport costs, distributional issues may provide a partial justification for the different tax treatment of diesel and gasoline cars in several European countries. Indeed, quite a few countries (e.g., Belgium, Germany, Italy) impose higher annual vehicle taxes on diesel cars but lower fuel taxes on diesel in comparison with gasoline cars (see ACEA (1996)). To the extent that diesel cars are used relatively more by high demand drivers and that the latter are assigned a lower weight in the objective function the model suggests a higher fixed tax on diesel than on gasoline cars, and 
correspondingly a lower variable tax. Second, pricing of public transport may be consistent with the optimal tax rules. Although public fares are typically highly complex and a variety of alternative options are available, in many European countries standard fares consist of a fixed fee, independent of distance, plus a distance-related tariff. Moreover, for specific customer types (low income families, children, large family size, etc.) social rates are typically available that imply a price reduction that, in percentage terms, rises with distance (NMBS (1996)). This implicitly implies that a relatively large price reduction is provided on the distance-related component. To the extent that the government is interested in redistribution towards high demand users of the public transport system (i.e., a positive $\gamma$ ) this pricing behavior is consistent with the prescriptions of the model. Indeed, under ceteris paribus conditions the social tariff would imply a reduction in the variable price.

\subsection{Extensions and potential applications}

Several final remarks are in order. First, although the derivation of the results of this paper were facilitated by assuming a logit specification to model the consumer's discrete choice, our findings are directly applicable to other discrete choice models as well. Generalisation to an arbitrary distribution of the random utility components is discussed in Appendix C. There we show that all results of this paper generally apply to a very broad class of discrete choice models. Second, note that this paper may have useful implications for the design of numerical optimal taxation models for specific markets such as the car market. The typical approach in the applied literature is to either ignore the distinction between fixed and variable taxes (see, e.g., Mayeres and Proost (1997)) or to model the demand for durables and the associated fixed and variable 
costs in a highly simplified way. For example, Conrad and Schroder (1991) and Koopman (1995) suggest to distinguish committed and noncommitted consumption and to allocate fixed costs entirely to the former. It is clear that explicit incorporation of the discrete choice process into numerical optimisation models provides a promising alternative to these approaches. Third, the model could be extended to include externalities. This may be highly policy-relevant. For example, in the case of car taxation it may make sense to subsidise specific car types for environmental reasons. A first effort in that direction is described in De Borger and Swysen (1997). They analyse two-part tariffs in the presence of externalities in a simple representative consumer model, thereby ignoring heterogeneity and distributional issues. Moreover, an empirical application to optimal car taxation in Belgium is provided. It is shown that even under these simplifying assumptions the optimal variable tax rule is quite complex and depends on the type of externality considered. A crucial parameter in the optimal tax rules turns out to be the impact of the externality on the ownership probabilities.

\section{Conclusion}

In this paper we presented a simple model of welfare-optimal two-part pricing within the framework of a model of discrete choice. It was shown that the pricing structure strongly depends on the price sensitivity of the entry probabilities, on the elasticities of conditional demands, on the degree and the nature of heterogeneity between consumers, and on the nature and degree of the government's distributional concerns. In the absence of distributional concerns, 
the optimal tax rule for the variable fee boils down to a multidimensional generalisation of results in Schmalensee (1981), Brown and Sibley (1986), and Laffont and Tirole (1993). The sign and magnitude of the variable tax specifically reflects the nature and degree of consumer heterogeneity in conditional demands as well as in the price sensitivities of the participation probabilities. It was shown that in the absence of collection or other fixed costs associated with participation, the fixed fee may be negative. The sign of the participation fee was shown to be positive as long as an increase in the variable fee implies a reduction in average conditional demand. The analysis furthermore generalised the 'corrected' Ramsey rule and provided an explicit and intuitive relation between the Ramsey correction term and the sign of the (net of collection costs) fixed fee. If the government does care about distribution, the results indicated that depending on the nature and degree of distributional preferences either the fixed or variable fee may increase. 


\section{Acknowledgments}

I am grateful to Fred Schroyen for detailed written suggestions on a previous version of this paper, to Wilfried Pauwels and Frank Verboven for stimulating discussions, and to two anonymous referees for many critical remarks.

\section{References}

ACEA, 1996. Motor vehicle taxation in Europe. Association of European Automobile Manufacturers, Brussels.

Anderson, S.P., De Palma, A., Thisse, J.,1993. Discrete choice theory of product differentiation. MIT Press, Cambridge, MA.

Ben Akiva, M., Lerman, S.R.,1987. Discrete choice analysis: Theory and application. MIT Press, Cambridge, MA.

Börsch-Supan, A., 1987. Econometric analysis of discrete choice: with applications to the demand for housing in the US and West Germany. Springer Verlag, Berlin.

Breautigam, R.R., 1989. Optimal policies for natural monopolies. In: R. Schmalensee and R. Willig (Eds), The Handbook of Industrial Organisation, Volume II. Elsevier Science Publishers, North Holland, New York, 1246-1289.

Brown, S.J., Sibley, D.S., 1986. The theory of public utility pricing. Cambridge University Press, Cambridge.

Conrad, K., Schröder, M., 1991. Demand for durable and nondurable goods, environmental policy and consumer welfare. Journal of Applied Econometrics 6, 271-286.

Cremer, H., Gahvari, F., 1995. Nonlinear pricing, redistribution and optimal tax policy. Paper presented at the 51st Congress of the International Instritute of Public Finance, Lisbon, Portugal. 
Damus, S., 1981. Two-part tariffs and optimum taxation: the case of railway rates. American Economic Review 71, 65-79.

De Borger, B., Swysen, D., 1997. Optimal two-part tariffs in the presence of externalities. Paper presented at the 53st Congress of the International Instritute of Public Finance, Kyoto, Japan.

De Borger, B., Ochelen, S., Proost, S., Swysen, D., 1997. Alternative transport pricing and regulation policies: A welfare analysis for Belgium in 2005. Transportation Research D vol. 2, 177-198.

De Jongh, G.C., 1990. An indirect utility model of car ownership and private car use. European Economic Review 34, 971-985.

Diamond, P., 1975. A many-person Ramsey tax rule. Journal of Public Economics 4, 227-244.

Dubin, J., McFadden, D.L., 1984. An econometric analysis of residential electric appliance holdings and usage. Econometrica 52, 345-362.

Feldstein, M.S., 1972. Equity and efficiency in public sector pricing: the optimal two-part tariff. Quarterly Journal of Economics 86, 175-187.

Glazer, A., Niskanen, E.,1992. Parking Fees and Congestion. Regional Science and Urban Economics 22, 123-132.

Koopman, G.J., 1995. Policies to reduce CO2 emissions from cars in Europe: A partial equilibrium analysis. Journal of Transport Economics and Policy 29, 53-70.

Laffont, J.J., Tirole, J., 1993. A theory of incentives in procurement and regulation. MIT Press, Cambridge, MA.

Leland, H.E., Meyer, R.A., 1976. Monopoly pricing structures with imperfect information. Bell Journal of Economics 7, 449-462. 
Mayeres, I., Proost, S., 1997. Optimal tax and public investment rules for congestion type of externalities. Scandinavian Journal of Economics 99, 261-279.

Manski, C.F., 1977. The structure of random utility models. Theory and Decision 8, 229-254.

Ng, Y.-K., Weisser, M., 1974. Optimal pricing with a budget constraint: the case of the two-part tariff. Review of Economic Studies 41, 337-345.

NMBS, 1996, Annual Report. National Railroad Company, Brussels.

Rosen, H.S., 1979. Housing decisions and the US income tax: An econometric analysis. Journal of Public Economics 11, 1-23.

Phlips, L., 1983. The Economics of Price Discrimination. Cambridge University Press, Cambridge.

Schmalensee, R., 1981. Monopolistic two-part pricing arrangements. Bell Journal of Economics $8, \quad 445-467$.

Spence, A.M., 1980. Multi-product quantity-dependent prices and profitability constraints. Review of Economic Studies 47, 821-841.

Sharkey, W., Sibley, D., 1993. Optimal nonlinear pricing with regulatory preferences over consumer type. Journal of Public Economics 50, 197-229.

Small, K.A., 1983. The incidence of congestion tolls on urban highways. Journal of Urban Economics 13, 90-111.

Small, K.A., Rosen, H.S., 1981. Applied welfare economics with discrete choice models. Econometrica 49, 105-130.

Train, K., 1986. Qualitative choice analysis: Theory, econometrics, and an application to automobile demand. MIT Press, Cambridge, MA.

Train, K., Ben-Akiva, M., Atherton, T., 1989. Consumer preferences and self selecting tariffs. Review of Economics and Statistics 71, 62-73. 
Train; K., McFadden, D.L., Ben-Akiva, M., 1987. The demand for local telephone services: a fully disaggregated model of residential calling patterns and services choice. Rand Journal of Economics 18 Spring, 109-123.

Wilson, R., 1993. Nonlinear Pricing. Oxford University Press, Oxford. 


\section{Appendix A: Derivation of the optimal two-part tariff}

In this appendix we provide more details on the derivation of the pricing rules reported in the main body of the paper. Recall the welfare optimisation problem

$$
\begin{aligned}
& \operatorname{Max}_{p, F} \sum_{h} \beta^{h} n^{h} \mu^{h} \boldsymbol{\Psi} \mathbf{p}\left(\frac{V_{1}^{h}}{\mu^{h}}\right)+\exp \left(\frac{V_{2}^{h}}{\mu^{h}}\right) \\
& \text { s.t. } \quad(p-c) \overline{\mathbf{F}} n^{h} \pi^{h} x^{h} \mathbf{k} F
\end{aligned}
$$

where the following shorthand notation is used: $V_{1}^{h}=V_{1}^{h}\left(p, y^{h}-F\right), \quad V_{2}^{h}=V_{2}^{h}\left(y^{h}\right)$, $\pi^{h}=\pi^{h}\left(p, y^{h}-F\right)$ and $x^{h}=x^{h}\left(p, y^{h}-F\right)$. The first-order condition with respect to $\mathrm{p}$ can be written as

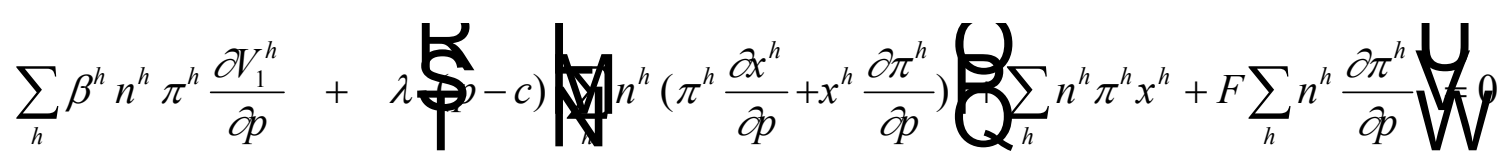

It will be instructive for the interpretation to decompose price effects associated with conditional demand into a Slutsky substitution effect and an income effect using the Slutsky equation

$$
\frac{\partial x^{h}}{\partial p}=\frac{\partial \hat{x}^{h}}{\partial p}-\frac{\partial x^{h}}{\partial y^{h}} x^{h}, \text { where } \quad \hat{x}^{h}=\hat{x}^{h}\left(p, F, u^{h}\right) \text { is the income-compensated }
$$

demand function. The above Slutsky relation follows from differentiation of the definition $\hat{x}^{h}\left(p, F, u^{h}\right)=x^{h}\left(p, e^{h}\left(p, F, u^{h}\right)-F\right)$, where $e^{h}($.$) is the expenditure function for a type h-$ individual. An analogous decomposition can be applied with respect to the probability functions $\pi^{h}$ (see Small and Rosen (1981)). Defining compensated choice probabilities $\hat{\pi}^{h}$ and noting that by definition $\hat{\pi}^{h}=\hat{\pi}^{h}\left(p, F, u^{h}\right)=\pi^{h}\left(p, e^{h}\left(p, u^{h}\right)-F\right)$, we obtain

$$
\frac{\partial \pi^{h}}{\partial p}=\frac{\partial \hat{\pi}^{h}}{\partial p}-\frac{\partial \pi^{h}}{\partial y^{h}} x^{h}
$$


Using the various decompositions in the first-order condition yields

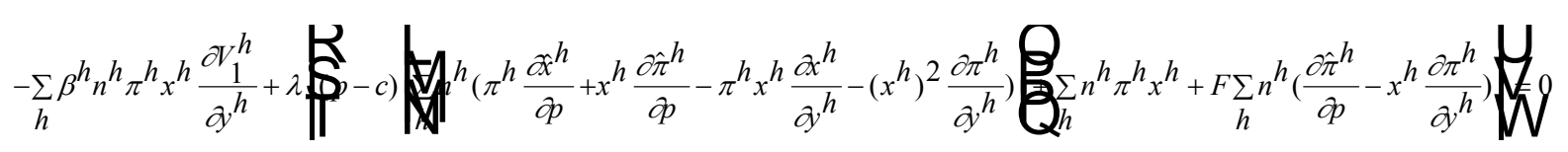

Separating the compensated price effects and the income effects yields, after some simple manipulations, equation (4) reported in Section 3 of the paper:

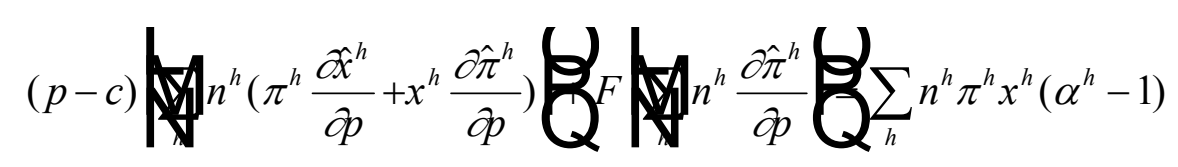

where

$$
\left.\alpha^{h}=\frac{\beta^{h}}{\lambda} \frac{\partial V_{1}^{h}}{\partial y^{h}}+\frac{1}{\pi^{h}} \mathbf{f}-c\right)\left(\pi^{h} \frac{\partial x^{h}}{\partial y^{h}}+x^{h} \frac{\partial \pi^{h}}{\partial y^{h}}\right)+F \frac{\partial \pi^{h}}{\partial y^{h}}
$$

The $\alpha^{h}$ have a clear interpretation in terms of Diamond's (1975) net marginal social utility of income, which expresses the marginal social utility of income in terms of government revenue. It is 'net' in the sense that it incorporates the induced tax payments due to the increase in income. It is easy to show that the $\alpha^{h}$ measure the net marginal social utility of income for buyers of the good (in other words, conditional on ownership), normalised by the appropriate probability of ownership. To see this, note by differentiation of the social welfare function that the marginal social utility of providing an increase in income $y^{h}$ to an owner of the good belonging to group $\mathrm{h}$ is given by

$$
\pi^{h} \beta^{h} \frac{\partial V_{1}^{h}}{\partial y^{h}}
$$

Dividing through by the shadow cost of public funds $\lambda$ expresses utility in units of government revenue. Moreover, the induced tax payments that an extra dollar of income to a consumer of the good in group $\mathrm{h}$ would generate is given by 


$$
(p-c)\left(\pi^{h} \frac{\partial x^{h}}{\partial y^{h}}+x^{h} \frac{\partial \pi^{h}}{\partial y^{h}}\right)+F \frac{\partial \pi^{h}}{\partial y^{h}}
$$

Adding these two terms and dividing by the probablity of ownership yields the interpretation given above.

Next briefly consider the first-order condition with respect to F. It is given by

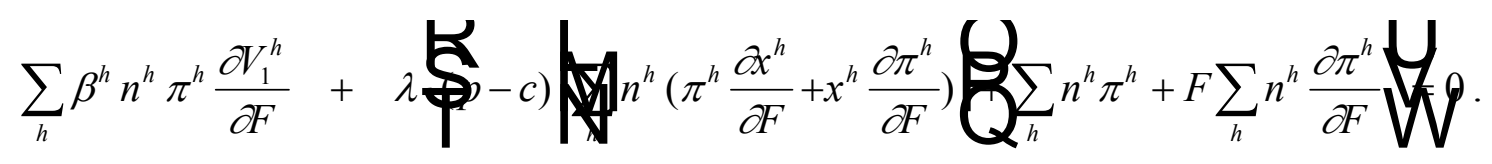

A similar procedure can be followed to rewrite this condition. First note that

$$
\frac{\partial V_{1}^{h}}{\partial F}=-\frac{\partial V_{1}^{h}}{\partial y^{h}} \text { and } \quad \frac{\partial x^{h}}{\partial F}=-\frac{\partial x^{h}}{\partial y^{h}}
$$

Moreover, differentiation of the logit probabilities with respect to $\mathrm{p}$ and $\mathrm{F}$ immediately shows that

$$
\frac{\partial \pi^{h}}{\partial F}=\frac{1}{x^{h}} \frac{\partial \pi^{h}}{\partial p}
$$

Using these expressions together with the above decompositions in substitution and income effects allows the condition for $\mathrm{F}$ to be written as follows

$$
(p-c) \sqrt{\mathbb{1}} n^{h} \frac{\partial \hat{\pi}^{h}}{\partial p} \mathbf{Q}_{F} \boldsymbol{W}^{h} \frac{1}{x^{h}} \frac{\partial \hat{\pi}^{h}}{\partial p} \mathbf{Q}_{h} n^{h} \pi^{h}\left(\alpha^{h}-1\right)
$$

This is equation (5) in the main body of the paper.

\section{$\underline{\text { Appendix B }}$}

The purpose of this appendix is to show that the term D defined in subsection 2.1 of the paper is positive. To see this, note that it can be written as 


$$
\left.D=\left(\sum_{h} n^{h} \pi^{h} \frac{\hat{x}^{h}}{\partial p}\right)\left(\sum_{h} \rho^{h}\right)+\mathbb{W} \rho^{h}\left(x^{h}\right)^{2} \rho^{h}\right)-\left(\sum_{h} \rho^{h} x^{h}\right)^{2} .
$$

Further manipulations yield

$$
D=\left(\sum_{h} n^{h} \pi^{h} \frac{\partial^{h}}{\partial p}\right)\left(\sum_{h} \rho^{h}\right)+\left(\sum_{h} \rho^{h}\right)^{2}
$$

where $\omega^{h}=\frac{\rho^{h}}{\sum \rho^{h}}$. Noting that this can be written as

$$
\begin{aligned}
& \sum_{k} \rho^{k} \\
& D=\left(\sum_{h} n^{h} \pi^{h} \frac{\hat{x}^{h}}{\partial p}\right)\left(\sum_{h} \rho^{h}\right)+\left(\sum_{h} \rho^{h}\right)^{2}\left[\operatorname{var}_{\omega}(x)\right]
\end{aligned}
$$

immediately implies $\mathrm{D}>0$, since both terms on the right-hand side are necessarily positive.

\section{Appendix C: Generalisation to other discrete choice models}

The logit model was used in the main body of the paper to study the optimal two-part tariff in a discrete choice framework. The logit specification yields a simple closed-form solution for the objective function of the optimal taxation problem (i.e., the maximum of the expected value of conditional utilities over alternatives can be written as a simple closed-form expression) and therefore highly simplified the derivation of the results. In this appendix we show, however, that the results presented in the paper all apply in general to other discrete choice models as well. To see this, note that the general formulation of the optimal taxation problem can be written as 


$$
\begin{aligned}
& \underset{p, F}{\operatorname{Max}} \sum_{h} \beta^{h} n^{h} \mathbf{O}\left[\operatorname{Max}\left(v_{1}^{h}, v_{2}^{h}\right)\right] \mathbf{t} \\
& \text { s.t. } \quad(p-c) \text { } n^{h} \pi^{h} x^{h} \mathbf{k} F n^{h} \pi^{h} \mathbf{k}=R \text {, }
\end{aligned}
$$

where $E[]$ is the expected value operator, and

$$
\begin{aligned}
& v_{1}^{h}=V_{1}^{h}+u_{1}^{h} \\
& v_{2}^{h}=V_{2}^{h}+u_{2}^{h}
\end{aligned}
$$

Although for many other discrete choice models (e.g., the probit model often used in empirical work), neither the probabilities $\pi^{h}$ nor the expected value of maximum utility over alternatives can be written in closed-form, one can easily show that the derivative of $E\left[\operatorname{Max}\left(v_{1}^{h}, v_{2}^{h}\right)\right]$ with respect to deterministic utility $V_{1}^{h}$ associated with participation is given by the participation probability $\pi^{h}$. Formulating the Lagrangean and differentiating with respect to $\mathrm{p}$ and $\mathrm{F}$ then yields first-order conditions that are identical to those derived in appendix $\mathrm{A}$, and the analysis can proceed completely analogously.

To show the above statement let us ignore the superscript h to simplify notation. Assume that the stochastic utility components are i.i.d. with density $\mathrm{g}($.$) and distribution function \mathrm{G}($.$) . An$ individual will participate if $v_{1}>v_{2}$ or, alternatively, if $u_{2}<V_{1}-V_{2}+u_{1}$. In other words, the participation probability conditional on a given value for $u_{1}$ is simply given by $G\left(V_{1}-V_{2}+u_{1}\right)$. Using this observation allows us to write

$$
E\left[\operatorname{Max}\left(v_{1}, v_{2}\right)\right]=Z_{g}\left(u_{1}\right) V^{V_{2}+u_{1}}\left(V_{1}+u_{1}\right) g\left(u_{2}\right) d u_{2}+Z_{1_{1}-v_{2}+u_{1}}\left(V_{2}+u_{2}\right) g\left(u_{2}\right) d u_{2}
$$

or, denoting the term between square brackets by $\mathrm{Z}$

$$
E\left[\operatorname{Max}\left(v_{1}, v_{2}\right)\right]=Z_{g}\left(u_{1}\right)[Z] d u_{1} \text {. }
$$


Differentiation yields

$$
\frac{\partial\left[E\left[\operatorname{Max}\left(v_{1}, v_{2}\right)\right]\right]}{\partial V_{1}}=Z_{-\infty} g\left(u_{1}\right) \frac{\partial Z}{\partial V_{1}} d u_{1}
$$

Now noting that

$$
\frac{\partial Z}{\partial V_{1}}=\frac{\partial \nabla_{1}^{V_{2}+u_{1}}\left(V_{1}+u_{1}\right) g\left(u_{2}\right) d u_{2}+\boldsymbol{Z}_{1-\gamma_{2}+u_{1}}\left(V_{2}+u_{2}\right) g\left(u_{2}\right) d u_{2}}{\partial V_{1}}
$$

and applying the rules for differentiation of integrals, we obtain

$$
\frac{\partial Z}{\partial V_{1}}=\left(V_{1}+u_{1}\right)\left[g\left(V_{1}-V_{2}+u_{1}\right)\right]+\left(Z^{V_{2}+u_{1}} g\left(u_{2}\right) d u_{2}\right)+\left[V_{2}+\left(V_{1}-V_{2}+u_{1}\right)\right]\left[g\left(V_{1}-V_{2}+u_{1}\right)\right](-1) .
$$

Rearranging yields

$$
\frac{\partial Z}{\partial V_{1}}=Z_{-\infty}^{V_{2}+u_{1}} g\left(u_{2}\right) d u_{2}=G\left(V_{1}-V_{2}+u_{1}\right) .
$$

Substitution in (C.1) gives

$$
\frac{\partial\left[E\left[\operatorname{Max}\left(v_{1}, v_{2}\right)\right]\right]}{\partial V_{1}}=Z_{-\infty} g\left(u_{1}\right) G\left(V_{1}-V_{2}+u_{1}\right) d u_{1} .
$$

The expression on the right-hand-side is just the unconditional ownership probability: it is obtained by integrating the probability of ownership, conditional on a given value of $u_{1}$. We finally have, therefore

$$
\frac{\partial\left[E\left[\operatorname{Max}\left(v_{1}, v_{2}\right)\right]\right]}{\partial V_{1}}=\pi
$$

In other words, the derivative of expected maximum utility with respect to the deterministic utility component is simply the participation probability. Moreover, differentiation of the probability with respect to $\mathrm{p}$ and F, respectively, and using Roy's identity immediately establishes that fact that 


$$
\frac{\partial \pi}{\partial F}=\frac{1}{x} \frac{\partial \pi}{\partial p}
$$

Using (C.2) and (C.3) in the first-order conditions of the general problem formulated in this appendix and following the steps explained in Appendix A leads to expressions that are identical to those derived for the logit formulation presented in the main body of the paper (see (4) and (5). All results immediately follow as in the text. 
Table 1: Relation between the nature of heterogeneity and the structure of the two-part tariff

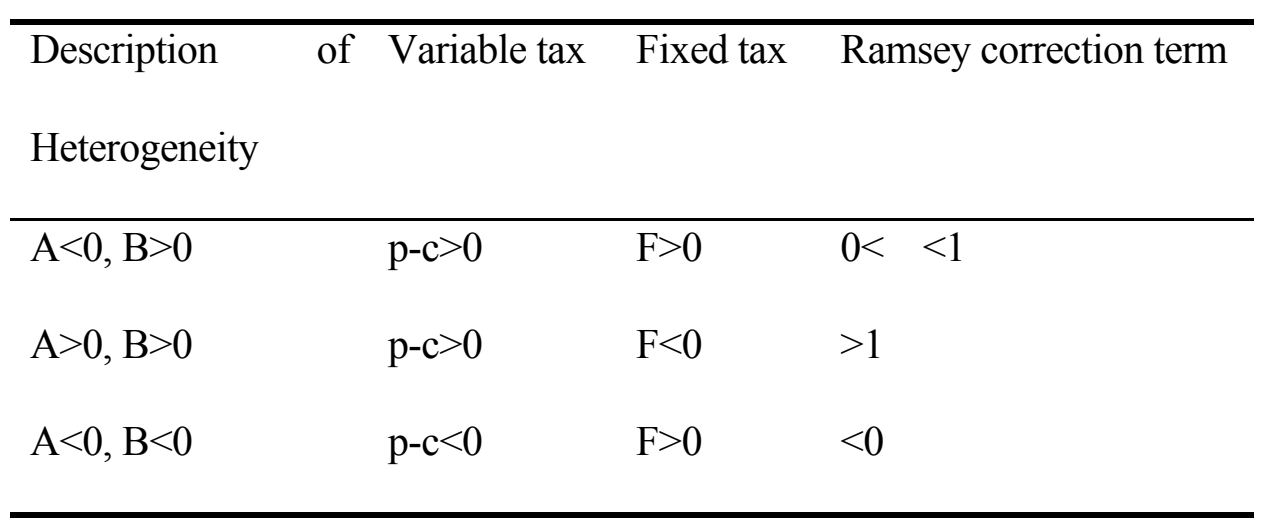

